SLAC-PUB-10089

\title{
Localized tachyon mass and a g-theorem analogue
}

\author{
Sang-Jin Sin \\ Stanford Linear Accelerator Center, Stanford University, Stanford CA 94305 * \\ and \\ Department of Physics, Hanyang University, Seoul, 133-791, Korea ${ }^{\dagger}$
}

October 30, 2018

\begin{abstract}
We study the localized tachyon condensation (LTC) of non-supersymmetric orbifold backgrounds in their mirror Landau-Ginzburg picture. Using he existence of four copies of $(2,2)$ worldsheet supersymmetry, we show at the CFT level, that the minimal tachyon mass in twisted sectors shows somewhat analogous properties of c- or g-function. Namely, $m:=\left|\alpha^{\prime} M_{m i n}^{2}\right|$ satisfies $m(M) \geq m\left(D_{1} \oplus D_{2}\right)=\max \left\{m\left(D_{1}\right), m\left(D_{2}\right)\right\}$. $c$ - $g$ - $m$ - functions share the common property $f(M) \geq f\left(D_{1} \oplus D_{2}\right)$ for $f=c, g, m$, although they have different behavior in detail.
\end{abstract}

${ }^{*}$ Work supported partially by the department of Energy under contract number DE-AC03-76SF005515.

${ }^{\dagger}$ Permanent address 


\section{Introduction}

The study of open string tachyon condensation[1] has led to many interesting consequences including classification of the D-brane charge by K-theory. While the closed string tachyon condensation involve the change of the background spacetime and much more difficult, if we consider the case where tachyons can be localized at the singularity, one may expect the maximal analogy with the open string case. Along this direction, the study of localized tachyon condensation (LTC) was considered first in 2] using the brane probe and renormalization group flow and by many others [3, 4, 5, 6, 7, 9]. The basic picture is that tachyon condensation induces cascade of decays of the orbifolds to less singular ones until the spacetime supersymmetry is restored. Therefore the localized tachyon condensation has geometric description as the resolution of the spacetime singularities.

Soon after, Vafa 3 considered the problem in the Landau-Ginzburg (LG) formulation using the Mirror symmetry and confirmed the result of [2]. In 4], Harvey, Kutasov, Martinec and Moore studied the same problem using the $\mathrm{RG}$ flow as deformation of chiral ring and in term of toric geometry. In both papers, the worldsheet $\mathrm{N}=2$ supersymmetry was utilized in essential ways.

The tachyon condensation process can be regarded as a RG-flow, along which there is a decreasing quantity, c-function, [10] for unitary conformal field theories. For the localized tachyon condensation in non-compact space, however, $c$ is constant 2, 4 and can not measure any dynamical process. Therefore it would be very interesting to have a quantity which has a property of monotonicity along the RG-flow. Along this line, the authors of [4] suggested a quantity, $g_{c l}$, which is a closed string analogue of the ground state degeneracy in open string theory[11]. On the other hand, Dabholkar and Vafa[5] suggested the maximal R-charge of Ramond sector (see [17, 18. for ealier study on this quantity), as the height of tachyon potential in the twisted sector describing the localized tachyon condensation. Although both suggestions are well motivated by physical intuitions, the prediction of two quantity are slightly different [7]. In [7], it was also suggested that the lowest twisted tachyon mass increases along RG flow. Using the spectral flow of $\mathrm{N}=2 \mathrm{CFT}$ and CTP invariance of the Ramond sector together with the charge-mass relation for chiral primaries, one can easily see that the proposal of [7] is equivalent to the GSO projected version of the one given by [5].

The monotonically increasing property of R-charge is related to a theorem in singularity theory called semi-continuity of spectrum[13] in singularity theory, which was conjectured by 
Arnold [12] and proved later by Varchenko[14] and Steenbrink[15]. These mathematical result can be applied [17] to the $N=2$ supersymmetric Landau-Ginzburg theory due to non-renormalization theorem. In our case, the LG theory that is mirror to the orbifold $\mathbb{C}^{r} / \mathbb{Z}_{n}$ is not an ordinary LG theory but an orbifolded LG [16] model whose chiral ring structure is very different from the ordinary LG and hence the theorem can not be applied directly. Although it is easy to see the monotonicity of R-charge for $\mathbb{C} / Z_{n}$ case, it is non-trivial for $\mathbb{C}^{2} / Z_{n}$ or higher dimensional cases.

The main goal of this paper is to prove that the lowest tachyon mass or equivalently the minimal $R$-charge in orbifold CFT and type 0 theories increases when we compare those in $U V$ and IR fixed points, which is conjectured in [5, 7]. As shown in [3, 4], the result of decay of $\mathbb{C}^{2} / Z_{n}$ is a sum of two disconnected theory and this fact introduces extra care when we compare with initial and final theories. When we denote the process of a mother theory decaying into two disconnected daughter theories by $M \rightarrow D_{1} \oplus D_{2}$, the c-function satisfies $c(M) \geq c\left(D_{1}\right)$ and $c(M) \geq c\left(D_{2}\right)$, while g-function seems to have $g(M) \geq g\left(D_{1}\right)+g\left(D_{2}\right)$ [4]. It turns out that for minimal tachyon mass, if we define $m=\left|\alpha^{\prime} M_{m i n}^{2}\right|$, it satisfies $m(M) \geq m\left(D_{1} \oplus D_{2}\right)=\max \left\{m\left(D_{1}\right), m\left(D_{2}\right)\right\}$, which is again different from both c- and g-function. Although $c$ - $g$ - $m$ - functions have different behavior in detail they share the common property

$$
f(M) \geq f\left(D_{1} \oplus D_{2}\right), \quad f=c, g, m .
$$

In order to avoid possible confusion and also to emphasize the difference with c-theorem, we call this monotonicity property of minimal tachyon mass as m-theorem.

Interestingly, our method applies only to the orbifolded Landau-Ginzburg thoery and does not apply to the generic LG theories. In this sense, our method is complementary to the method used in mathematical literature.

We use the mirror LG picture of Vafa and the existence of the many copies of $(2,2)$ worldsheet supersymmetries for $\mathbb{C}^{2} / \mathbb{Z}_{n}$. In $\mathrm{CFT}$ of $\mathbb{C}^{2} / Z_{n}$, there are $2^{2}$ extended chiral ring structures according to the choice of complex structures of each $\mathbb{C}$ factor. We call them as $c c, c a, a c$ and a a rings. For string spectrum, we need to put spectrum of all 4 sectors together. When we consider the behavior of $c c$ ring elements under the condensation of a tachyon in $c c$ ring, we can establish an explicit mapping between spectrum of initial and final orbifold conformal field theories. We can show that individual R-charge of tachyons increases under the process. This is possible since we have control over the RG-process due to the world sheet $(2,2)$ supersymmetry off the criticality, which povide the non-renormalization theorem. However, what happen to the R-charges of operators in $c a$ or other rings when a tachyon operator in $c c$ ring condensate? The answer is that we lose control, since we lose all supersymmetry off the criticality hence we do not 
have non-renormalization theorem.

What saves us from this difficulty is the presence of the enhanced $2^{r}$ copies of $(2,2)$ worldsheet SUSY in orbifold CFT's. This is because its presence allows us to choose the supersymmetry generators $G_{-\frac{1}{2}}^{ \pm}$and complex structure such that the condensing tachyon belongs to $c c$-ring. We can then determine the generators of the daughter theories. Since we know that the final products of the decay are again orbifold theories [2, 3, 4, knowing the fate of the $c c$-ring element is enough to establish the fate of entire spectrum. Using this special property of orbifold theories, we will be able to establish linear mappings for each of 4 chiral rings. ${ }^{1}$ We can also show that the linear mapping has the property such that the $\mathrm{R}$-charges of their images are bigger than the R-charges of the originals. The mere existence of such mappings will enable us to show our main goal: the minimal charge increases under TC.

In section 2, we give a brief summary of mirror LG model. In section 3, we give the proof of the statement and we conclude in section 4.

\section{Mirror symmetry and Orbifolds}

In this subsection we give a summary of Vafa's work [3] on localized tachyon condensation. The orbifold $\mathbb{C}^{r} / Z_{n}$ is defined by the $\mathbb{Z}_{n}$ action given by equivalence relation

$$
\left(X_{1}, \ldots, X_{r}\right) \sim\left(\omega^{k_{1}} X_{1}, \ldots, \omega^{k_{r}} X_{r}\right), \quad \omega=e^{2 \pi i / n} .
$$

We call $\left(k_{1}, \cdots, k_{r}\right)$ as the generator of the $\mathbb{Z}_{n}$ action. The orbifold can be imbedded into the gauged linear sigma model(GLSM) [19]. The vacuum manifold of the latter is described by the D-term constraints

$$
-n\left|X_{0}\right|^{2}+\sum_{i} k_{i}\left|X_{i}\right|^{2}=t
$$

Its $t \rightarrow-\infty$ limit corresponds to the orbifold and the $t \rightarrow \infty$ limit is the $O(-n)$ bundle over the weighted projected space $W P_{k_{1}, \ldots, k_{r}}$. $X_{0}$ direction corresponds to the non-compact fiber of this bundle and $t$ plays role of size of the $W P_{k_{1}, \ldots, k_{r}}$.

By dualizing this GLSM, we get a LG model with a superpotential[20]

$$
W=\sum_{i=0}^{r} \exp \left(-Y_{i}\right)
$$

\footnotetext{
${ }^{1}$ Some of these mapping does not describe tachyon condensation process of individual R-charges. They just connect between the spectrum of mother and daughter theories.
} 
where twisted chiral fields $Y_{i}$ are periodic $Y_{i} \sim Y_{i}+2 \pi i$ and related to $X_{i}$ by $\operatorname{Re}\left[Y_{i}\right]=\left|X_{i}\right|^{2}$. Introducing the variable $u_{i}:=e^{-Y_{i} / n}$, the D-term constraint is expressed as $e^{-Y_{0}}=e^{t / n} \prod_{i} u^{k_{i}}$. The periodicity of $Y_{i}$ imposes the identification : $u_{i} \sim e^{2 \pi i / n} u_{i}$ which necessitate modding out each $u_{i}$ by $\mathbb{Z}_{n}$. The result is usually described by

$$
\left[W=\sum_{i=1}^{r} u_{i}^{n}+e^{t / n} \prod_{i} u^{k_{i}}\right] / /\left(\mathbb{Z}_{n}\right)^{r-1} .
$$

which describe the mirror Landau-Ginzburg model of the linear sigma model. As a $t \rightarrow-\infty$ limit, mirror of the orbifold is

$$
\left[W=\sum_{i=1}^{r} u_{i}^{n}\right] / /\left(\mathbb{Z}_{n}\right)^{r-1} .
$$

Since it is not ordinary Landau-Ginzburg theory but an orbifolded version, the chiral ring structure of the theory is very different from that of LG model. For example, the dimension of the local ring of the super potential is always $n-1$, regardless of $r$.

We list some properties of orbifolded LG theory for later use.

The true variable of the theory are $Y_{i}$ not $u_{i}$ related by $u_{i}=e^{-Y_{i} / n}$. As a consequence, monomial basis of the chiral ring is given by

$$
\left\{u_{1}^{p_{1}} u_{2}^{p_{2}} \mid\left(p_{1}, p_{2}\right)=\left(n\left\{j k_{1} / n\right\}, n\left\{j k_{2} / n\right\}\right), j=1, \ldots, n-1\right\},
$$

and $u_{1}^{p_{1}} u_{2}^{p_{2}}$ has weight $\left(p_{1}, p_{2}\right)$ and charge $\left(p_{1} / n, p_{2} / n\right)$.

- It has been known (see for example [8], for $\mathbb{C}^{2} / \mathbb{Z}_{n}$, any worldsheet fermion generated tachyon can be constructed as a BPS state, i.e, a member of a chiral ring. as a consequence of existence of 4 copies of $(2,2)$ worldsheet SUSY for this special theory.

- It is convenient to consider the weight of a state as sum of contribution from each complex plane. For example, the weight of $u_{1}^{n a_{1}} u_{2}^{n a_{2}}$ can be considered as sum of $a_{1}$ from $u_{1}$ and $a_{2}$ from $u_{2}$. $\left(a_{1}, a_{2}\right)$ form a point in the weight space. As we vary $j$ in $a_{i}=\left\{j k_{i} / n\right\}$, the trajectory of the point in weight space will give us a parametric plot in the plane.

- The weight space is a lattice in torus of size $n \times n$. The identification of weights by modulo $n$ corresponds to shifting string modes. However, periodicity of the generator $\left(k_{1}, k_{2}\right)$ is $2 n$ and $\left(k_{1}, k_{2}\right)$ and $\left(k_{1}, k_{2}+n\right)$ do not generate the same theory in general. We choose the standard range of $k_{i}$ between $-n+1$ to $n-1$. This is because the GSO projection depends not only the R-charge vector $\left(\left\{j k_{1} / n\right\},\left\{j k_{2} / n\right\}\right)$ but also the G-parity number $G=\left[j k_{1} / n\right]+\left[j k_{2} / n\right]$. We will comeback to this when we discuss the GSO projection. 
- When $n$ and $k_{i}$ are not relatively prime, we have a chiral primary whose R-charge vector is $(p / n, 0)$. We call this as the reducible case and eliminate from our interests. This is a spectrum that is not completely localized at the tip of the orbifold. Sometimes, even in the case we started from non-reducible theory, a tachyon condensation leads us to the reducible case.

\section{3 m-theorem}

The conformal weight in the NS-sector is related to the mass by [21, 22, 23]

$$
\frac{1}{4} \alpha^{\prime} M^{2}=\Delta-\frac{1}{2}
$$

For $\mathbb{C}^{1} / \mathbb{Z}_{n}$ model, $\Delta_{\text {min }}=1 / 2 n$ so that $\alpha^{\prime} M_{\text {min }}^{2}=-2(1-1 / n)$ is proportional to the deficit angle of the cone. The maximal R-charge in NS sector and the minimal tachyon mass is simply related. Let's imbed the orbifold into 8 dimensional transverse target space of lightcone string theory. Then the transverse spacetime is $\mathbb{C}^{r} / \mathbb{Z}_{n} \times R^{8-2 r}$. The ground states of the twisted sectors in NS sectors are chiral or anti-chiral primary and the charge is simply given by the weight $q= \pm 2 \Delta$. By the spectral flow, $q_{R}=q_{N S}-\hat{c} / 2$, we have $\frac{1}{4} \alpha^{\prime} M^{2}=\frac{1}{2}\left(q_{R}+\frac{\hat{c}}{2}\right)-\frac{1}{2}$. and

$$
\alpha^{\prime} M_{\min }^{2}=Q_{\min }^{5}+\hat{c}-2
$$

where 2 in $Q_{\text {min }}^{5}=2 q_{R \text {,min }}$ comes from summing left and right R-charges. Using the CPT symmetry on the Ramond sector, we have $q_{R}^{\min }=-q_{R}^{\max }$. Therefore above statement can also be written as

$$
\max \left|\alpha^{\prime} M^{2}\right|=Q_{\max }^{5}+2-\hat{c}:=m
$$

The main goal of this paper is to show that $m$ has a property that is a reminiscent of a c-function:

$$
m(U V) \geq m(I R)
$$

We call this as a m-theorem to prevent possible confusion with c-theorem or g-theorem.

\section{1 proof of the theorem}

This work is heavily dependent on the result of companion paper [8], where details of transition of spectrum is discussed. Our interest is how the spectrum flows under the RG-flow. In lack of of control of off-shell theory, it is in general difficult question to address. However, spectrum 
of UV and IR theories are readily available since both are conformal field theory. Since any tachyon generated by worldsheet fermion can be thought as a chiral ring element in one choice of $(2,2)$ SUSY, we can assume, without loss of generality, that the condensing tachyon with weight $p=\left(p_{1}, p_{2}\right)$ is an element of $c c$-ring. Then consider other element in the same $c c$-ring whose weight is $q=\left(q_{1}, q_{2}\right)$. For definiteness, let's say $q \in \Delta_{-}$in the notation of [8]. The R-charge of it is $R_{q}=\left(q_{1}+q_{2}\right) / n$. Now after the condensation of $p=\left(p_{1}, p_{2}\right), q$ is moved to $q^{\prime}=T_{p}^{-}(q)$ [ where,

$$
T_{p}^{-}=\left(\begin{array}{cc}
p_{2} / n & -p_{1} / n \\
0 & 1
\end{array}\right)
$$

whose R-charge is

$$
R_{q^{\prime}}=\left(q_{2}-p \times q / n\right) / p_{2} .
$$

If $p$ represents a tachyonic state, it must be below the diagonal. Namely,

$$
p_{1}+p_{2} \leq n
$$

Therefore the difference in the R-charge between before and after the process is given by

$$
R_{q^{\prime}}-R_{q}=\left(n-p_{1}-p_{2}\right) q_{2} / n p_{2} \geq 0
$$

The same inequality holds for $q \in \Delta_{+}$. For $p, q \in a c$ ring, we can apply the same argument by replacing

$$
p \rightarrow \bar{p}=\left(p_{1}, n-p_{2}\right), \quad q \rightarrow \bar{q}=\left(q_{1}, n-q_{2}\right) .
$$

Therefore we arrive at the result:

Lemma: The R-charge of a relevant chiral primary operator increases under condensation of tachyon in the same ring.

The eq. (3.8) also shows that under the condensation of marginal operator, there is no change in R-charge of any operator. Due to the mass-charge relation discussed before, we can make the same statements for the tachyon mass. The above statement shows that any of the spectrum is a candidate of the c-function of the twisted sector. However, this statement does not exclude the possibility of level crossing. That is, the ordering of the R-charge can be changed during the process.

What happen to the R-charges of operators in $c a$ ring when a tachyon in $c c$ ring condensate? The answer is that we lose control, since we lose the world sheet $(2,2)$ super symmetry off the criticality and we lose non-renormalization theorem. In fact if one naively apply the mapping 
$T_{p}^{ \pm}$to the $c a$-ring elements, we get non-integer power of $u_{i}$ 's. Similarly, when we condense a $c a$ ring element, we lose control over the $c c$ ring spectrum.

However, when an element in ca ring turn on, we have control over other ca ring elements instead. It is holomorphic and protected by worldsheet SUSY $G_{c a}^{+}$. Since we have choice of selecting complex structure in each plane independently, we can choose any combination of complex structure to define the holomorphic co-ordinate of $\mathbb{C}^{2}$. We can call $u_{1}, \bar{u}_{2}$ as the holomorphic co-ordinates just as we can call $u_{1}, u_{2}$ as a holomorphic coordinate. As far as other combination does not enter in the theory, operators are protected by the worldsheet supersymmetry.

As a warm up, we first consider the case where the minimal charge of initial and final theories belong to $c c$-ring, so that we do not need to consider other chiral rings. Let $q_{0}$ denote the a state of minimal R-charge, namely,

$$
R\left[q_{0}\right] \leq R[q], \text { for all } q .
$$

We want to compare the minimal charge of the initial charge and that of the final state. Let $q^{\prime}$ be a minimal charge of a final theory. There are two theories in the final states and one choose any of it, say up-theory. Then $q^{\prime}$ should come from a $q \in \Delta_{+}$such that $q^{\prime}=T_{p}^{+}(q)$. Due to the monotonicity of R-charge, we have $R\left[q^{\prime}\right]>R[q]$. On the other hand, $R[q]$ can not be smaller than $R\left[q_{0}\right]$, by definition of $q_{0}$. Therefore we have inequality

$$
R\left[q_{\min }^{\text {initial }}\right]<R\left[q_{\text {min }}^{\text {final }}\right] .
$$

The same inequality holds for the down-theory as well.

Some of the relevant operators, which are precisely those in the triangle $\triangle B P A$, will be pushed out to irrelevant operator after $\mathrm{P}$ is condensed. One may worry about the converse possibility that some irrelevant operators of the initial theories flow to the relevant operator. Following lemma tells us that it does not happen.

Lemma : Relevant chiral primary states of final theory comes only from the relevant ones in the initial theory.

Proof: Let $q^{\prime} / p_{2}$ be the charge of a relevant operator in the down-theory and $q$ be its pre-image in the original theory, i.e, $q^{\prime}=T_{p}^{-}(q)$. Our question is whether $q_{1}^{\prime}+q_{2}^{\prime}<p_{2}$ implies $q_{1}+q_{2}<n$ or not. This can be answered simply by calculating the inverse of $T_{p}^{-}$.

$$
q=\left(T_{p}^{-}\right)^{-1}\left(q^{\prime}\right)=\frac{n}{p_{2}}\left(\begin{array}{cc}
1 & p_{1} / n \\
0 & p_{2} / n
\end{array}\right)\left(\begin{array}{l}
q_{1}^{\prime} \\
q_{2}^{\prime}
\end{array}\right)=\left(\begin{array}{c}
\left(n q_{1}^{\prime}+p_{1} q_{2}^{\prime}\right) / p_{2} \\
q_{2}^{\prime}
\end{array}\right)
$$

Now,

$$
q_{1}+q_{2}=\left(n q_{1}^{\prime}+q_{2}^{\prime}\left(p_{1}+p_{2}\right)\right) / p_{2} \leq n\left(q_{1}^{\prime}+q_{2}^{\prime}\right) / p_{2}<n
$$


Following is an easy consequence.: Minimal $R$-charge of the cc ring increases under condensation of tachyon in cc-ring. More explicitly,

$$
\min _{l=1}^{n-1}\left(\left\{l k_{1} / n\right\}+\left\{l k_{2} / n\right\}\right)
$$

increases under tachyon condensation.

So far is story of $c c$-ring. We need to consider all chiral rings together. So we are interested in the behavior of the R-charge which is smallest in the union of $c c$ ring and $c a$-ring. To do this, we reconsider the problem of the fate of $c a$-ring under the condensation of tachyon in $c c$-ring. Although we do not have any control over the flow of the ca ring spectrum, we know what is the final theory and its total set of the spectrum. We ask whether any tachyon mass of the final theory can be considered as an image of some mapping with the property of R-charge increasing. To do this we want to show that there is a map that takes the some of chiral ring of the mother theory to $c a$ or $a c$ ring of the daughter theories. Notice that, in general, the $c a$ ring of $n\left(k_{1}, k_{2}\right)$ is $c c$ ring of $n\left(k_{1},-k_{2}\right)$ and the daughter theory has structure $p_{1}\left(k_{1}, p \times k / n\right) \oplus p_{2}\left(-p \times k / n, k_{2}\right)$. First, the $c a$ ring of the daughter theory $p_{1}\left(k_{1}, p \times k / n\right)$ is $c c$-ring of $p_{1}\left(k_{1},-p \times k / n\right)$ which is expected to be the image of the $c c$ ring of $n\left(k_{1},-k_{2}\right)$ under some mapping $F_{p}^{+}$, which is not necessarily associated with physical process. It turns out that $F_{p}^{+}$can be chosen as $T_{\left(p_{1},-p_{2}\right)}^{+}$:

$$
F_{p}^{+}(\bar{q}):=T_{p^{\prime}}^{+}(\bar{q})=\left(q_{1}, p_{1}-p \times q / n\right)
$$

where $\bar{q}=\left(q_{1}, n-q_{2}\right) \in c a$ ring and $p^{\prime}=\left(p_{1},-p_{2}\right)$. One can check that

$$
R\left[F_{p}^{+}(\bar{q})\right]>R[\bar{q}] \text { if } \bar{q} \in \text { ca ring } .
$$

Similarly, the $a c$ ring of the daughter theory $p_{2}\left(-p \times k / n, k_{2}\right)$ is $c c$ ring of $p_{2}\left(p \times k / n, k_{2}\right)$, which can be considered as the image of the $c c$ ring of $n\left(-k_{1}, k_{2}\right)$ by the map $F_{p}^{-}$defined by

$$
\left.F_{p}^{-}(\tilde{q}):=T_{-p^{\prime}}^{-}\left(n-q_{1}, q_{2}\right)\right)=\left(p_{2}+p \times q / n, q_{2}\right)
$$

where $\tilde{q}=\left(n-q_{1}, q_{2}\right) \in a c$ ring. It can be also shown that

$$
R\left[F_{p}^{-}(\tilde{q})\right]>R[\tilde{q}] \text { if } \tilde{q} \in \text { ac ring }
$$

Now let $q_{0}^{\prime}$ be the tachyon with lowest mass in the daughter theory. Let it belong to the up-theory. Then we can without loss of generality assume that it belongs to ca ring due to the equivalence $c a$ and $a c$ ring in their spectrum. (If it belongs to $c c$ ring, we have shown already 
what we want to show.) Then $q^{\prime}=F_{p}^{+}(\bar{q})$ for some $\bar{q}$, which has bigger charge than the minimal charge of initial theory. Using the property of eq. (3.16),

$$
R\left[q^{\prime}\right] \geq R[q] \geq R\left[q_{0}\right]
$$

as desired. Similarly, if $q^{\prime}$ belongs to down theory, we can assume that it belongs to the $a c$-ring of the down theory. Then Then $q^{\prime}=F_{p}^{-}(\tilde{q})$ for some $\tilde{q}$, which has bigger charge than the minimal charge of initial theory $q_{0}$. Using the property of eq. (3.18),

$$
R\left[q^{\prime}\right] \geq R[\tilde{q}] \geq R\left[q_{0}\right]
$$

as desired.

Therefore we proved following: In the conformal field theory of orbifolds, the minimal $\mathrm{R}$ charge of the final theory is bigger than that of the initial theory under the condensation of a tachyon generated by a world sheet fermion;

$$
\min _{l=1}^{n-1}\left[\min \left(\left\{l k_{1} / n\right\}+\left\{l k_{2} / n\right\}-1,\left\{l k_{1} / n\right\}-\left\{l k_{2} / n\right\}\right)\right]
$$

increases when we compare its value in the initial and final theories. Equivalently, in terms of absolute values of tachyon mass,

$$
m(I R) \geq m(U V)
$$

A few remarks are in order:

- These theorems are world sheet fact. The same statement conjectured in [7] is the GSO projected version for which we need to take into account the GSO projection. However, tachyons in NS-NS sector is not projected out by the type 0 GSO projection, so the above conclusion is true in type 0 string theory level. For type II we will discuss in detail in next subsection.

- There are two independent theories in the final stage of tachyon condensation. Each theory will have its own minimal charges. We should take the smaller of the two, since the minimal mass of final theory is the minimal over all final spectra. Namely, the minimal R-charges of two theories in final stage are not to be added to compare with the initial one, contrary to the treatment of $g_{c l}$ in [4].

- From the discussion of previous subsection, this is the proof of the conjecture stated by Dabholkar and Vafa in [5]. The R-charge here is that in NS sector. The R-charge of Ramond 
sector is related to that of NS sector by spectral flow. Since there are CPT invariance in Ramond sector, the statement that minimal charge increases is equivalent to statement that maximal charge decreases.

\section{Discussion and Conclusion}

In this paper, we have studied the localized condensation in non-supersymmetric orbifold using the $(2,2)$ world sheet SUSY in mirror LG picture. We study the localized tachyon condensation in Mirror Landau-Ginzburg picture as well as the toric geometry picture of non-supersymmetric orbifold backgrounds. Due to the two copies of $(2,2)$ worldsheet supersymmetry, any worldsheet fermion generated tachyon can be considerred as a BPS state. Utilizing this fact, we showed that the R-charge of chiral primaries increases under the process of localized tachyon condensation. The minimal tachyon mass in twisted sectors increases in CFT and type 0 string and plays similar role of the c- or g-function in the twisted sectors. By working out how the individual chiral primaries are mapped under the tachyon condensation, we have proved that R-charges of chiral primaries increase under tachyon condensation. We studied the GSO projection and found that in many aspects, the separate notion of type II and type 0 is not preserved under the tachyon condensation.

We emphasized similarity of c-,g- and $\mathrm{m}$ - functions in the introduction. Here we want to draw the reader's attention to one more object; the orbifold Witten index $\mu=n$, counting the number of (un)twisted sector. Under the transition $n(1, k) \rightarrow p_{1}(1, s) \oplus p_{2}(-s, k)$, $\mu$ obviously satisfy the relation

$$
\mu(M) \geq \mu\left(D_{1}\right)+\mu\left(D_{2}\right)
$$

from the very fact that $\left(p_{1}, p_{2}\right)$ is a tachyon. This property is similar to the g-function. Notice, however, for marginal case $\mu(M)=\mu\left(D_{1}\right)=\mu\left(D_{2}\right)$ does not hold for witten index unlike the c-function. This is just parallel to the case of minimal R-charge. Inspite of its simplicity, we did not use this as an semi-c-object to study orbifold decay for following reasons;

- It does not depends on the generator $k$. Too many theories have the same witten index. Therefore it is not a sharp indicator.

- It can not be defined off-shell. It is true topological index that is independent of finite deformation of the theory. Therefore it can not be used for full study of RG-flow. The minimal tachyon mass, on the other hand, can be defined off-shell along the line of [17, 18]. 
We now discuss the limitation and related future works. First of all, our work is confined to orbifold fixed points before and after the tachyon condensation. It would be interesting to work out the detail of the off-shell. One may ask what is the geometry for the finite condensation co-efficient of LG in terms of gauged linear sigma model? A work related to this question has appeared [24]. Another related work is [25], where the Bondi energy [26] as a c-function was discussed based on the earlier work by Tseytlin [27. The use of this work, however, seems to be rather limited for studying orbifold transitions, since it requires that the asymptotic regions be invariant under the transition, which can not be fulfilled by the orbifolds.

Secondly, we considered the case where only one tachyon generated by a worldsheet fermion and the method of this paper does not work if we consider simultaneous condensation of two tachyons in the different chiral rings.

Thirdly, our work is mostly about CFT and type 0 theory rather than type II theory. For type II theory, there is only one way by which the theorem can be broken, namely, if the minimal charge of mother theory is projected out by the GSO and the minimal charge of the daughter theory is kept, then it may happen that the minimal charge of the daughter theory is smaller than that of the mother theory. It is very plausible that such possibility happens. Surprisingly, however, in all example we considered, the tachyon condensation that cause such possibility is forbidden due to GSO projection in all the examples we looked at so far. We wish to come back to this issue in later publication.

\section{Acknowledgement}

I would like to thank Lance Dixon, Michael Gutperle, Shamit Kachru, Amir Kashani-Poor, Matthias Klein and Michael Peskin for discussions, and especially to Eva Silverstein for her support and interests, and to Allan Adams for his collaboration in the initial stage. This work is partially supported by the Korea Research Foundation Grant (KRF-2002-013-D00030). 


\section{References}

[1] A. Sen, "Non-BPS states and branes in string theory," arXiv:hep-th/9904207, and references citing it; For review, see

A. Sen, "Stable non-BPS states in string theory," JHEP 9806, 007 (1998) arXiv:hep-th/9803194, and references therein.

[2] A. Adams, J. Polchinski and E. Silverstein, "Don't panic! Closed string tachyons in ALE space-times," JHEP 0110, 029 (2001) arXiv:hep-th/0108075.

[3] C. Vafa, "Mirror symmetry and closed string tachyon condensation," arXiv:hep-th/0111051.

[4] J. A. Harvey, D. Kutasov, E. J. Martinec and G. Moore, "Localized tachyons and RG flows," arXiv:hep-th/0111154.

[5] A. Dabholkar and C. Vafa, "tt* geometry and closed string tachyon potential," arXiv:hep-th/0111155.

[6] A. Dabholkar, "Tachyon condensation and black hole entropy," Phys. Rev. Lett. 88, 091301 (2002) arXiv:hep-th/0111004.

[7] Sang-Jin Sin, "Tachyon mass, c-function and Counting localized degrees of freedom," Nucl. Phys. B637 (2002)395, arXiv:hep-th/0202097.

[8] S. J. Sin, "Comments on the fate of localized tachyons"

[9] T. Takayanagi and T. Uesugi, "Orbifolds as Melvin geometry," arXiv:hep-th/0110099. Y. Michishita and P. Yi, "D-brane probe and closed string tachyons," Phys. Rev. D 65, 086006 (2002) arXiv:hep-th/0111199,

Soonkeon Nam and Sang-Jin Sin, "Condensation of Localized Tachyons and Spacetime Supersymmetry", arXiv:hep-th/0201132

R. Rabadan and J. Simon, "M-theory lift of brane-antibrane systems and localised closed string tachyons," JHEP 0205, 045 (2002) arXiv:hep-th/0203243;

A. M. Uranga, "Localized instabilities at conifolds," arXiv:hep-th/0204079

T. Sarkar, "Brane probes, toric geometry, and closed string tachyons," Nucl. Phys. B 648, 497 (2003) arXiv:hep-th/0206109. T. Suyama, "Closed string tachyons and RG flows," JHEP 0210, 051 (2002) arXiv:hep-th/0210054; 
J. L. Barbon and E. Rabinovici, "Remarks on black hole instabilities and closed string tachyons," arXiv:hep-th/0211212,

Y. H. He, "Closed string tachyons, non-supersymmetric orbifolds and generalised McKay correspondence," arXiv:hep-th/0301162.

[10] A. B. Zamolodchikov, "Irreversibility' Of The Flux Of The Renormalization Group In A 2-D Field Theory," JETP Lett. 43, 730(1986) [Pisma Zh. Eksp. Teor. Fiz. 43, 565 (1986)].

[11] I. Affleck and A. W. Ludwig, "Universal Noninteger 'Ground State Degeneracy' In Critical Quantum Systems," Phys. Rev. Lett. 67, 161 (1991).

[12] V.I.Arnol'd, "On some problems in singularity theory", In Geometry and Analysis, Papers dedicated to V.K.Patodi, 1-9(1981).

[13] V.I. Arnol'd, Sinularity theory (Selected papers), Cambridge University Press 1981, V.I. Arnol'd(Ed), Dynamical systems VI, Springer.

[14] A.N. Varchenko, "Semi-continuity of the spectrum and an upper bound for the number of singular points of a projective hypersurface", Soviet. math., Dolk.27, 735-739 (1983)

[15] J.H.M.Steenbrink, "Semicontinuity of the singularity spectrum". Invent. Math. 79, 557566.(1985)

[16] C. Vafa, "String Vacua And Orbifoldized L-G Models," Mod. Phys. Lett. A 4, 1169 (1989); K. A. Intriligator and C. Vafa, "Landau-Ginzburg Orbifolds," Nucl. Phys. B 339, 95 (1990).

[17] S. Cecotti and C. Vafa, "Topological antitopological fusion," Nucl. Phys. B 367, 359(1991).

[18] S. Cecotti, P. Fendley, K. A. Intriligator and C. Vafa, "A New supersymmetric index," Nucl. Phys. B 386, 405 (1992) arXiv:hep-th/9204102.

[19] E. Witten, "Phases of $\mathrm{N}=2$ theories in two dimensions," Nucl. Phys. B 403, 159 (1993) arXiv:hep-th/9301042.

[20] K. Hori and C. Vafa, "Mirror symmetry," arXiv:hep-th/0002222.

[21] L. J. Dixon, D. Friedan, E. J. Martinec and S. H. Shenker, "The Conformal Field Theory Of Orbifolds," Nucl. Phys. B 282, 13 (1987). 
[22] A. Dabholkar, "Strings on a cone and black hole entropy," Nucl. Phys. B 439, 650 (1995) arXiv:hep-th/9408098.

[23] D. A. Lowe and A. Strominger, "Strings near a Rindler or black hole horizon," Phys. Rev. D 51, 1793(1995) arXiv:hep-th/9410215.

[24] E. J. Martinec and G. Moore, "On decay of K-theory," arXiv:hep-th/0212059,

E. J. Martinec, "Defects, decay, and dissipated states," arXiv:hep-th/0210231

[25] M. Gutperle, M. Headrick, S. Minwalla and V. Schomerus, "Space-time energy decreases under world-sheet RG flow," JHEP 0301, 073 (2003) arXiv:hep-th/0211063.

[26] H.Bondi, M. Van der Burg and A. Metzner, "Gravitational waves in general relativity VII. Waves from axi-symmetric isolated systems"' Proc. Roy. Soc. London, Ser. A269,21(1962).

[27] A. A. Tseytlin, "Conditions of Weyl invariance of two-dimensional sigma model from equations of stationarity of central charge action," Phys. Lett. B 194, 63 (1987). 